\title{
Expression of microRNA-181 determines response to treatment with azacitidine and predicts survival in elderly patients with acute myeloid leukaemia
}

\author{
ALEKSANDRA BUTRYM ${ }^{1,2}$, JUSTYNA RYBKA ${ }^{1}$, DAGMARA BACZYŃSKA ${ }^{3}$, \\ RAFAŁ PORĘBA ${ }^{4}$, GRZEGORZ MAZUR ${ }^{4}$ and KAZIMIERZ KULICZKOWSKI ${ }^{1}$
}

\author{
Departments of ${ }^{1}$ Haematology, Blood Neoplasms and Bone Marrow Transplantation, and ${ }^{2}$ Physiology, \\ Wroclaw Medical University, Wroclaw 50-367; ${ }^{3}$ Department of Forensic Medicine, Molecular Techniques Unit, \\ Wroclaw Medical University, Wroclaw 50-345; ${ }^{4}$ Department of Internal and Occupational Diseases, \\ Hypertension and Clinical Oncology, Wroclaw Medical University, Wroclaw 50-556, Poland
}

Received December 2, 2015; Accepted June 2, 2016

DOI: $10.3892 / 01.2016 .4970$

\begin{abstract}
MicroRNAs (miRs) are small non-coding RNAs that play important roles in cell differentiation and survival. Abnormal expression of miRs has been demonstrated in numerous types of cancer, including acute myeloid leukaemia (AML). The aim of the present study was to evaluate miR-181 expression at diagnosis and following the completion of chemotherapy in AML patients, with regard to clinical response and outcome, particularly in patients treated with azacitidine. miR-181 expression was analysed using reverse transcription-quantitative polymerase chain reaction in 95 bone marrow specimens from newly diagnosed AML patients and in 20 healthy subjects for comparison. The results revealed upregulated miR-181 expression in the total cohort of AML patients, which was correlated with longer survival. However, in a subset of older AML patients treated with azacitidine, low miR-181 expression at diagnosis was a predictor for complete remission and prolonged survival. The findings indicated that miR-181 has an important role in AML and determines response to azacitidine treatment in older AML patients.
\end{abstract}

\section{Introduction}

Acute myeloid leukaemia (AML) is a haematological malignancy with a heterogeneous clinical course. Currently, gene expression profiling and various mutations (NPM1, CEBPA, FLT3-ITD) are used to stratify AML patients and distinguish

Correspondence to: Dr Aleksandra Butrym, Department of Haematology, Blood Neoplasms and Bone Marrow Transplantation, Wroclaw Medical University, Wybrzeże Ludwika Pasteura 4, Wroclaw 50-367, Poland

E-mail: aleksandra.butrym@gmail.com

Key words: miR-181, expression, acute myeloid leukemia, azacitidine, remission, response, survival prognostic subgroups (1). The standard of care for fit AML patients remains intensive chemotherapy based on anthracycline and cytarabine regimens, while in elderly populations, new therapeutic strategies are being tested (2-6). Azacitidine, a chemical nucleoside analogue, appears to be a promising option for elderly patients (7-9). Unfortunately, a large proportion of patients do not respond to AML therapy, while others will relapse in a short period of time. In the context of these data, new diagnostic and prognostic biomarkers are required for the appropriate selection of patients suitable for chemotherapy regimens and novel target therapies.

MicroRNAs (miRs) are a large family of non-coding small RNAs that are involved in many crucial processes, including cell differentiation, proliferation and apoptosis (10-12). Dysregulation of numerous miRs has been reported in solid tumours and haematological malignancies. Certain miRs have been identified to act as a tumour suppressors or oncogenes $(12,13)$. Furthermore, miRs are able to regulate the expression of many target genes through mRNA interference.

The miR-181 family has been previously studied in haematological malignancies (14-19). Human miR-181 genes are located on chromosomes 1, 9 and 19 (14). Members of miR-181 group (miR-181a, miR-181b, miR-181c and miR-181d) have been conserved during evolution, which may suggest that they are functionally important. Previous evidence has suggested an association between miR-181a expression and French-American-British (FAB) AML subtypes (20).

The purpose of the current study was to evaluate miR-181a expression in AML patients prior to chemotherapy compared with healthy controls, and miR-181 expression after chemotherapy was completed. The results were analysed with regard to the clinical features of AML patients and the type of induction chemotherapy (intensive vs. low-dose).

\section{Materials and methods}

Patient characteristics. This study included 95 patients [mean age \pm standard deviation (SD), 60.2 \pm 15.0 years; 
age range 22-90 years; male, 61\%] with newly diagnosed AML. Samples of the bone marrow for miR-181 expression analysis were collected prior to the start of chemotherapy and repeated following the completion of induction chemotherapy (40 patients). Patients were treated in the Department of Haematology, Blood Neoplasms and Bone Marrow Transplantation of Wroclaw Medical University (Wroclaw, Poland). A control group of 20 healthy subjects was also assessed [mean age $\pm \mathrm{SD}, 64.2 \pm 10.5$ years; age range, $39-80$ years; male, $65 \%$ ). According to the AML FAB classification, 7 patients had AML M0, 34 had M1, 29 had M2, 14 had M4 and 11 had M5. There were 73 patients with primary leukaemia and 22 patients with leukaemia secondary to myelodysplastic or myeloproliferative syndromes. A summary of patient characteristics is present in Table I.

Induction chemotherapy. Following diagnosis, 56 patients were treated with standard induction intensive chemotherapy (daunorubicin plus cytarabine 3+7), 27 received low-dose chemotherapy (low-dose cytarabine or azacitidine) and 12 received best supportive care only. Following the completion of induction therapy, the response to treatment was evaluated. Complete remission (CR) was defined by the Cheson criteria (21). Bone marrow samples were also reevaluated for miR-181 expression in 40 patients. Patients were followed up for a median of 21 months (range, 1-40 months).

AML patients treated with azacitidine. There were 17 newly diagnosed AML patients who were not suitable for intensive chemotherapy and were treated with azacitidine (4 women and 13 men; age range, 65-90 years; median age, 75 years). Azacitidine was administered at a dose of $75 \mathrm{mg} / \mathrm{m}^{2}$ subcutaneously on days 1-7 of 28-day cycles. Patients received 1-35 cycles of azacitidine (median, 7 cycles). There were 6 patients who achieved CR, 6 who had partial response or disease stabilisation and 5 who did not respond to therapy. At the time of analysis, all patients, except one, had succumbed to the disease.

Compliance with ethical standards. Research was conducted in compliance with the Declaration of Helsinki. Approval for the study was obtained from the Bioethics Committee of Wroclaw Medical University. Written informed consent for the study was obtained from all participants.

Isolation and expression analysis of miRs. Bone marrow mononuclear cells were isolated by Ficoll-Hypaque density gradient centrifugation. Total RNA and miRs were extracted from collected AML mononuclear cells using the mirVana ${ }^{\mathrm{TM}}$ miRNA Isolation Kit (Ambion; Thermo Fisher Scientific, Inc., Waltham, MA, USA) according to the protocol of the manufacturer. Subsequently, $5 \mu$ l of total miRNA was used as a template for the synthesis of cDNA using TaqMan MicroRNA Transcription Reaction Kit (Applied Biosystems; Thermo Fisher Scientific, Inc.) and $3 \mu \mathrm{l}$ of specific miRNA primers from the TaqMan MicroRNA Assays (Applied Biosystems; Thermo Fisher Scientific, Inc.). Individual reactions were conducted in a $15 \mu \mathrm{l}$ total volume with the following thermal condition: $16^{\circ} \mathrm{C}$ for $30 \mathrm{~min}, 42^{\circ} \mathrm{C}$ for $30 \mathrm{~min}$
Table I. Clinical characteristics of patients with acute myeloid leukaemia $(\mathrm{n}=95)$.

\begin{tabular}{lc}
\hline Characteristic & Value \\
\hline Gender, $\mathrm{n}$ & \\
Male & 56 \\
Female & 39 \\
Age (years) & \\
Range & $22-90$ \\
Median & 61 \\
French-American-British subtype, n & \\
M0 & 7 \\
M1/M2 & 63 \\
M4/M5 & 25 \\
White blood cells, x10 $/ 1$ & \\
Range & $0.2-295$ \\
Median & 14
\end{tabular}

Median

Median

Median

Median

$<50 \%$

Cytogenetics, $\mathrm{n}$

Favorable 5

Intermediate

Unfavorable

Chemotherapy, n

Intensive

Low-dose

Best supportive care

Mutation status, $\mathrm{n}$

AML/ETO (positive/negative) $\quad$ 4/56

CBFb-MYH11 (positive/negative) 2/58

NPM1 (positive/negative)

FLT3/ITD (positive/negative)

Complete remission, $\mathrm{n}$

Yes (total)

Yes (after first-line therapy)

No

Duration of remission, months

Range

Median

Time to relapse, months

Range

Median

Survival, months

Range

Median 


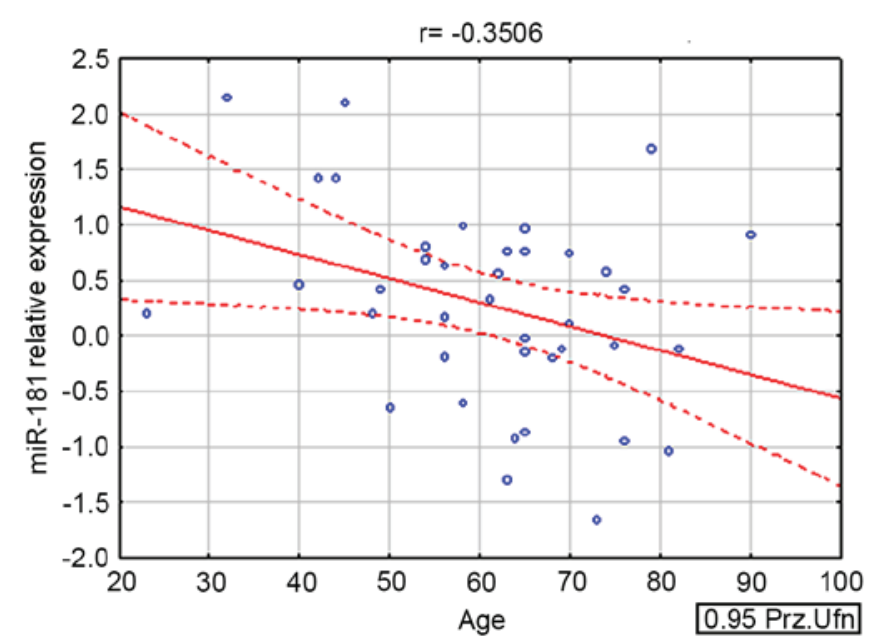

Figure 1. Indirect correlation between baseline miR-181 relative expression and patient age (years).

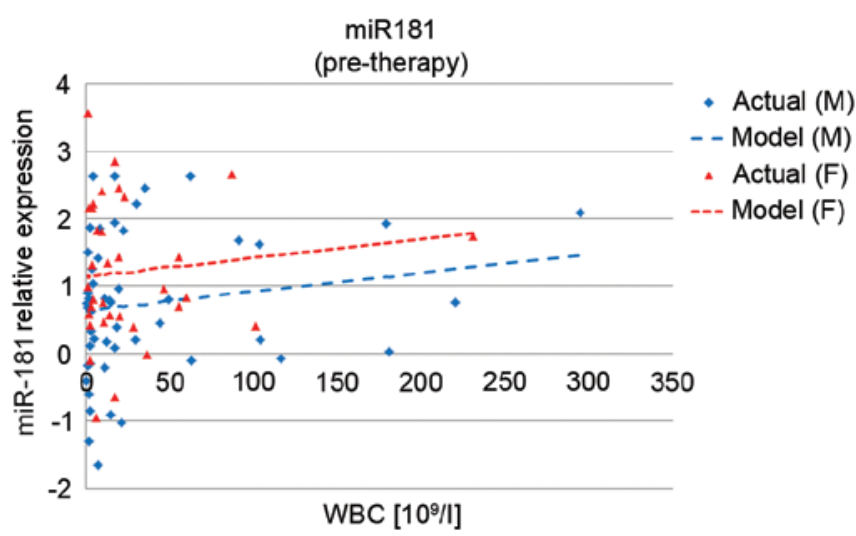

Figure 2. Correlation between miR-181 relative expression and gender. miR-181 expression was higher in F patients and was correlated with a higher WBC count. M, male; F, female; WBC, white blood cell.

and $85^{\circ} \mathrm{C}$ for $5 \mathrm{~min}$. TaqMan MicroRNA Assays for miR-181 (hsa-miR-181) and RNU48 were used. The expression levels of each miR were measured by the relative quantitative polymerase chain reaction (qPCR) method using TaqMan Gene Expression Assays and TaqMan Fast Universal PCR Master Mix (Applied Biosystems; Thermo Fisher Scientific, Inc.). All reactions were performed in triplicate in a total volume of $20 \mu \mathrm{l}$ on 96-well plates. qPCR was performed on 7900HT Fast Real-Time PCR System (Applied Biosystems; Thermo Fisher Scientific, Inc.) under the following thermal cycling conditions: $20 \mathrm{sec}$ at $95^{\circ} \mathrm{C}$; and 40 cycles of $1 \mathrm{sec}$ at $95^{\circ} \mathrm{C}$ and $20 \mathrm{sec}$ at $60^{\circ} \mathrm{C}$. For quantification, the samples were normalised against the expression of RNU48 miR. Relative quantification factors for the examined miRs were calculated using the $\Delta \Delta$ Cq method (22).

Statistical analysis. The differences in the mean levels of gene expression between the study and control patients were assessed using the Student's $t$-test (for independent samples). To examine the time to mortality or remission, a Cox regression analysis was applied (23). The differences between the gene expression levels before and after treatment were analysed

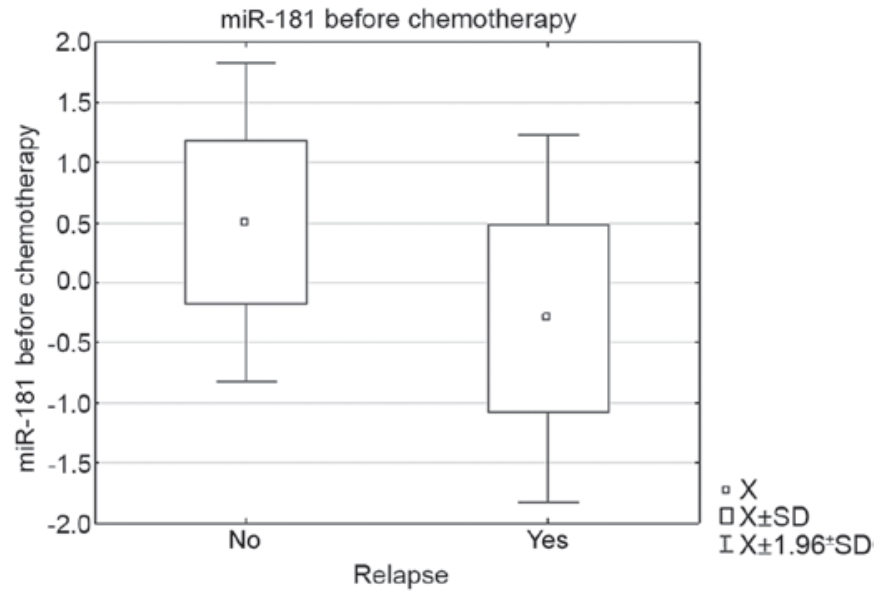

Figure 3. Correlation between baseline miR-181 relative expression and response to induction chemotherapy. Patients with higher miR-181 expression had a better prognosis and lower probability of relapse. $\mathrm{X}$, mean; $\mathrm{SD}$, standard deviation.

using robust regression and multivariate approach (24). The computation was performed in R software (25) and based on the simulation technique known as Gibbs sampling in WinBUGS platform (26). Kaplan-Meier survival curves were used to determine any significant relationship between miR-181 expression and clinical outcome. $\mathrm{P}<0.05$ indicated a statistically significant difference.

\section{Results}

miR-181 expression and clinical characteristics. miR-181 expression was found to be significantly higher in AML patients compared to the control group ( $\mathrm{P}=0.019)$, and was also indirectly correlated with the age of the patient $(\mathrm{r}=-0.350563$; $\mathrm{P}=0.032$ ) (Fig. 1). miR-181 expression was correlated with higher white blood cell count $(\mathrm{P}=0.0069)$. It was also associated with gender and was significantly higher in women than in men ( $\mathrm{P}=0.001)$ (Fig. 2).

Patients with higher miR-181 expression at diagnosis had better prognosis than those with lower miR-181 expression $(\mathrm{P}=0.048)$. In the group of AML patients who relapsed following remission, the mean miR-181 expression level at diagnosis was lower than in the group that maintained $\mathrm{CR}$ $(-0.299657$ vs. $0.500855 ; \mathrm{P}=0.012)$ (Fig. 3). The change in miR-181 expression following chemotherapy did not influence patients' clinical outcomes $(\mathrm{P}=0.065)$.

miR-181 expression and azacitidine therapy. At the time of AML diagnosis, miR-181 expression was correlated with the response to azacitidine treatment: Patients who responded to azacitidine therapy had lower miR-181 expression at diagnosis than those without remission $(-0.21$ vs. 1.26; $\mathrm{P}=0.02$ ) (Fig. 4).

Furthermore, miR-181 expression level prior to therapy determined patients' clinical outcomes. Its expression was indirectly correlated with survival after azacitidine therapy $(\mathrm{r}=-0.503172 ; \mathrm{P}=0.048)$; lower expression determined longer survival (median survival 22 vs. 5 months). On multivariate analysis, low miR-181 expression at diagnosis was an 


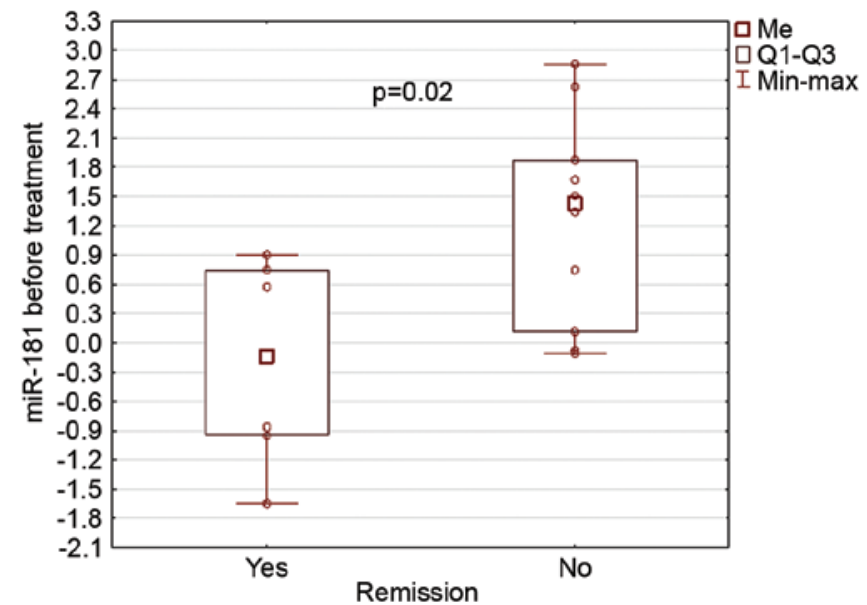

Figure 4. Correlation between baseline miR-181 expression and complete remission after chemotherapy with azacitidine. Me, median; Q1, first quartile; Q3, third quartile; min, minimum; max, maximum.

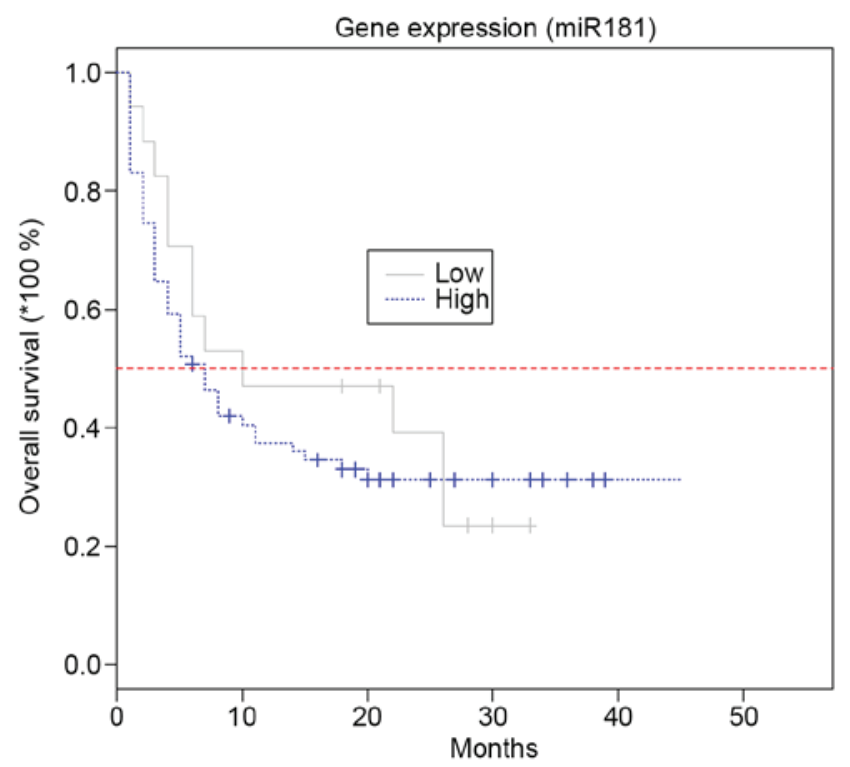

Figure 5. Estimated overall survival of acute myeloid leukaemia patients treated with azacitidine depending on miR-181 expression prior to chemotherapy.

independent predictive factor for response to azacitidine and prolonged survival ( $\mathrm{P}=0.012$; Fig. 5).

\section{Discussion}

Currently, an increasing number of studies on miRs in solid tumours and haematological malignancies are being conducted, with which our knowledge regarding the role of miRs in pathogenesis and the clinical course of the disease is still increasing. Dysregulation of the expression of various miRs is associated with the development and progression of AML (20,27-30).

The important role of miR-181 in AML has been already described in previous studies. Isken et al (31) and Debernardi et al (20) reported elevated expression of miR-181 in M1 and M2 FAB AML subtypes, while in M4 and M5 patients the miR-181, expression was lower. In the present study, higher levels of miR-181 expression were identified in the AML group compared to the control group; however, no association between expression and FAB AML subtype was observed. In addition, the current results suggest an indirect correlation of miR-181 expression with the age of AML patients. Furthermore, higher miR-181 expression was observed in women and associated with white blood cell count.

The role of miR-181 as a potential prognostic marker has been demonstrated in particular groups of AML subsets. A study by Li et al (16) revealed downregulation of miR-181 expression in cytogenetically abnormal AML cases, while its increased expression was associated with better prognosis. The results of the present study are in line with previously published studies, such as the study by Schwind et al (32) who confirmed a higher CR rate, and longer overall survival and disease-free survival times in patients with higher miR-181 expression levels. In the present study, lower miR-181 expression at diagnosis was associated with a higher risk of disease relapse.

Although high expression of miR-181 was observed in the total AML patient population and was correlated with better survival, low expression of miR-181 was observed in an older population treated with the hypomethylating drug azacitidine and was associated with prolonged survival. The novelty of our results relates to the relationship between miR-181 expression and response to azacitidine therapy in the subset of elderly patients with AML. In the aforementioned patient population, characterised by poor overall survival, no standard chemotherapy options are available. Azacitidine is a cytidine analogue which, in low doses, causes DNA demethylation by inactivation of the DNA methyltransferase-1 enzyme (33). Recently, it has been shown that azacitidine is an effective and safe induction therapy for elderly AML patients who are not suitable to undergo intensive treatment (34). However, there is still a group of patients who do not benefit from hypomethylating therapy.

To the best of our knowledge, the present study is the first to show a correlation between miR-181 expression and azacitidine efficacy in AML patients; lower expression was an independent predictor for good response to treatment and prolonged survival. With regard to the feasibility of miR-181 expression measurement, it is an easy tool in the selection of particular patient groups potentially benefiting from this kind of treatment.

In summary, the current study confirmed the presence of upregulated miR-181 expression in AML patients. Furthermore, for the first time, the ability of miR-181 expression to determine response to azacitidine in elderly patients has been shown. In addition, miR-181 may be used as a stratification tool to qualify patients for this kind of treatment, and to select potential responders to azacitidine therapy. Nevertheless, these results must be validated in a larger population of AML patients.

\section{Acknowledgements}

This study was supported by the Wroclaw Medical University Young Scientist Grant (no. Pbmn140). 


\section{References}

1. Shivarov V and Bullinger L: Expression profiling of leukemia patients: Key lessons and future directions. Exp Hematol 42: 651-660, 2014.

2. Ross K, Gillespie-Twardy AL, Agha M, Raptis A, Hou JZ, Farah R, Redner RL, Im A, Duggal S, Ding F, et al: Intensive chemotherapy in patients aged 70 years or older newly diagnosed with acute myeloid leukemia. Oncol Res 22: 85-92, 2015.

3. Wang ES: Treating acute myeloid leukemia in older adults Hematology Am Soc Hematol Educ Program 2014: 14-20, 2014.

4. Burnett AK, Russell N, Hills RK, Panoskaltsis N, Khwaja A, Hemmaway C, Cahalin P, Clark RE and Milligan D: A randomised comparison of the novel nucleoside analogue sapacitabine with low-dose cytarabine in older patients with acute myeloid leukaemia. Leukemia 29: 1312-1319, 2015.

5. Mayer J, Arthur C, Delaunay J, Mazur G, Thomas XG, Wierzbowska A, Ravandi F, Berrak E, Jones M, Li Y and Kantarjian HM: Multivariate and subgroup analyses of a randomized, multinational, phase 3 trial of decitabine vs treatment choice of supportive care or cytarabine in older patients with newly diagnosed acute myeloid leukemia and poor- or intermediate-risk cytogenetics. BMC Cancer 14: 69, 2014.

6. Kantarjian HM, Thomas XG, Dmoszynska A, Wierzbowska A, Mazur G, Mayer J, Gau JP, Chou WC, Buckstein R Cermak J, et al: Multicenter, randomized, open-label, phase III trial of decitabine versus patient choice, with physician advice, of either supportive care or low-dose cytarabine for the treatment of older patients with newly diagnosed acute myeloid leukemia. J Clin Oncol 30: 2670-2677, 2012.

7. Ramos F, Thépot S, Pleyer L, Maurillo L, Itzykson R, Bargay J, Stauder R, Venditti A, Seegers V, Martínez-Robles V, et al: Azacitidine frontline therapy for unfit acute myeloid leukemia patients: Clinical use and outcome prediction. Leuk Res 39: 296-306, 2015.

8. Lao Z, Yiu R, Wong GC and Ho A: Treatment of elderly patients with acute myeloid leukemia with azacitidine results in fewer hospitalization days and infective complications but similar survival compared with intensive chemotherapy. Asia Pac J Clin Oncol 11: 54-61, 2015.

9. Huang J, Lyu H, Wang J and Liu B: MicroRNA regulation and therapeutic targeting of survivin in cancer. Am J Cancer Res 5 : 20-31, 2014.

10. Larson RA: Micro-RNAs and copy number changes: New levels of gene regulation in acute myeloid leukemia. Chem Biol Interact 184: 21-25, 2010.

11. Yahya SM and Elsayed GH: A summary for molecular regulations of miRNAs in breastcancer. Clin Biochem 48: 388-396, 2015.

12. Li YQ, Ren XY, He QM, Xu YF, Tang XR, Sun Y, Zeng MS, Kang TB, Liu N and Ma J: MiR-34c suppresses tumor growth and metastasis in nasopharyngeal carcinoma by targeting MET. Cell Death Dis 6: e1618, 2015.

13. Drebber U, Lay M, Wedemeyer I, Vallböhmer D, Bollschweiler E, Brabender J, Mönig SP, Hölscher AH, Dienes HP and Odenthal M: Altered levels of the onco-microRNA 21 and the tumor-supressor microRNAs 143 and 145 in advanced rectal cancer indicate successful neoadjuvant chemoradiotherapy. Int J Oncol 39: 409-415, 2011

14. Weng H, Lal K, Yang FF and Chen J: The pathological role and prognostic impact of miR-181 in acute myeloid leukemia. Cancer Genet 208: 225-229, 2015.

15. Jinlong S, Lin F, Yonghui L, Li Y, Li Y and Weidong W: Identification of let-7a-2-3p or/and miR-188-5p as prognostic biomarkers in cytogenetically normal acute myeloid leukemia. PLoS One 10 e0118099, 2015

16. Li Z, Huang H, Li Y, Jiang X, Chen P, Arnovitz S, Radmacher MD, Maharry K, Elkahloun A, Yang X, et al: Up-regulation of a HOXA-PBX3 homeobox-gene signature following down-regulation of miR-181 is associated with adverse prognosis in patients with cytogenetically abnormal AML. Blood 119: 2314-2324, 2012.
17. Chu B, Zhong L, Dou S, Wang J, Li J, Wang M, Shi Q, Mei Y and Wu M: miRNA-181 regulates embryo implantation in mice through targeting leukemia inhibitory factor. J Mol Cell Biol 7: $12-22,2015$.

18. Huang P, Ye B, Yang Y, Shi J and Zhao H: MicroRNA-181 functions as a tumor suppressor in non-small cell lung cancer (NSCLC) by targeting Bcl-2. Tumour Biol 36: 3381-3387, 2015.

19. Su R, Lin HS, Zhang XH, Yin XL, Ning HM, Liu B, Zhai PF, Gong JN, Shen C, Song L, et al: MiR-181 family: Regulators of myeloid differentiation and acute myeloid leukemia as well as potential therapeutic targets. Oncogene 34: 3226-3239, 2015.

20. Debernardi S, Skoulakis S, Molloy G, Chaplin T, Dixon-McIver A and Young BD: MicroRNA miR-181a correlates with morphological sub-class of acute myeloid leukaemia and the expression of its target genes in global genome-wide analysis. Leukemia 21: 912-916, 2007.

21. Cheson BD, Bennett JM, Kopecky KJ, Büchner T, Willman CL, Estey EH, Schiffer CA, Doehner H, Tallman MS, Lister TA, et al: Revised recommendations of the International Working Group for Diagnosis, standardization of response criteria, treatment outcomes and reporting standards for therapeutic trials in acute myeloid leukemia. J Clin Oncol 21: 4642-4649, 2003.

22. Livak KJ and Schmittgen TD: Analysis of relative gene expression data using real-time quantitative PCR and the 2(-Delta Delta C(T)) Method. Methods 25: 402-408, 2001.

23. Cox DR: Regression models and life-tables. J R Stat Soc Series B Stat Methodol 34: 187-220, 1972

24. Congdon P (ed): Robust Regression Methods. In: Applied Bayesian Modelling. Wiley, Chichester, pp118-126, 2003.

25. R Core Team: A language and environment for statistical computing. Version 3.0.3. 2014. Vienna: R Foundation for Statistical Computing, http://www.r-project.org/.

26. Spiegelhalter D, Thomas A, Best N and Lunn D: WinBUGS. Version 1.4.3. 2003. Cambridge: Imperial College School of Medicine \& Medical Research Council - Biostatistics Unit (www.mrc-bsu.cam.ac.uk/bugs/winbugs/), 2003.

27. Butrym A, Rybka J, Baczyńska D, Tukiendorf A, Kuliczkowski K and Mazur G: Expression of microRNA-331 can be used as a predictor for response to therapy and survival in acute myeloid leukemia patients. Biomark Med 9: 453-460, 2015.

28. Cammarata G, Augugliaro L, Salemi D, Agueli C, La Rosa M, Dagnino L, Civiletto G, Messana F, Marfia A, Bica MG, et al: Differential expression of specific microRNA and their targets in acute myeloid leukemia. Am J Hematol 85: 331-339, 2010.

29. Grenda A, Budzyński M and Filip AA: Biogenesis of microRNAs and their role in the development and course of selected hematologic disorders. Postepy Hig Med Dosw (Online) 67: 174-185, 2013 (In Polish)

30. Butrym A, Rybka J, Baczyńska D, Tukiendorf A, Kuliczkowski K and Mazur G: Low expression of microRNA-204 (miR-204) is associated with poor clinical outcome of acute myeloid leukemia (AML) patients. J Exp Clin Cancer Res 34: 68, 2015.

31. Isken F, Steffen B, Merk S, Dugas M, Markus B, Tidow N, Zühlsdorf M, Illmer T, Thiede C, Berdel WE, et al: Identification of acute myeloid leukaemia associated microRNA expression patterns. Br J Haematol 140: 153-161, 2008.

32. Schwind S, Maharry K, Radmacher MD, Mrózek K, Holland KB, Margeson D, Whitman SP, Hickey C, Becker H, Metzeler $\mathrm{KH}$, et al: Prognostic significance of expression of a single microRNA, miR-181a, in cytogenetically normal acute myeloid leukemia: A cancer and leukemia group B study. J Clin Oncol 28: 5257-5264, 2010.

33. Derissen EJ, Beijnen JH and Schellens JH: Concise drug review: Azacitidine and decitabine. Oncologist 18: 619-624, 2013.

34. Dombret H, Seymour JF, Butrym A, Wierzbowska A, Selleslag D, Jang JH, Kumar R, Cavenagh J, Schuh AC, Candoni A, et al: International phase 3 study of azacitidine vs conventional care regimens in older patients with newly diagnosed AML with $>30 \%$ blasts. Blood 126: 291-299, 2015. 